\title{
THE COMPOSITION OF THE BILE FOLLOWING THE RELIEF OF BILIARY OBSTRUCTION ${ }^{1}$
}

BY CARL H. GREENE, WALTMAN WALTERS AND CLYDE H. FREDRICKSON²

(From the Divisions of Medicine and Surgery, The Mayo Clinic, Rochester, Minn.)

(Received for publication April 4, 1930)

The significance of the liver, as the largest glandular organ in the body, and its paramount importance in certain phases of intermediary metabolism, contrasts strangely with the paucity of knowledge, at least in man, concerning its secretion, the bile. The characteristic biliary constituents, bilirubin, the bile acids and cholesterol have been isolated and identified chemically. Experimental work on animals, especially on dogs with permanent biliary fistulas, has indicated the multiplicity of factors, such as diet, climate, fasting, various chemical poisons, infections and exercise, which may affect either the volume or composition of the bile. In this connection, the earlier work of Bidder and Schmidt (1852), and of Stadelmann (1891, 1896) and, in recent years, that of Whipple (1922) and Rous (1925) and their collaborators has been of fundamental significance.

As a result of these studies it is now recognized that there may be great variations in the flow of bile from day to day. The secretion of bile pigment, on the other hand, continues at a fairly constant rate except for changes due to hemorrhage, anemia or other disturances in the metabolism of the blood pigments. In consequence, the concentrations of the bile pigments tends to vary inversely with the volume of the bile, and McMaster, Broun and Rous (1923), and Greene and Snell (1928) have emphasized the extreme variations in concentration which occasionally may be found. The intimate relation between the diet and the excretion of cholesterol in the bile has recently been

${ }^{1}$ Preliminary report given at the Twenty-second Annual meeting of the American Society for Clinical Investigation, Atlantic City, New Jersey, May 5, 1930. J. Clin. Invest. 1930, ix, 30.

${ }^{2}$ Fellow in Surgery, The Mayo Foundation. 
commented on by McNee (1914) and by McMaster (1924). Several factors combine to determine the excretion of bile acids: (1) an endogenous factor responsible for the relatively constant excretion of bile acids in the fasting dog which may possibly be related to the metabolism of body protein; (2) an exogenous factor, determined by the diet and especially concerned with the amount and character of the protein therein, and (3) an intestinal factor as a result of the absorption of bile acids from the bowel, with the resultant establishment of the enterohepatic circulation originally postulated by Schiff (1870); this intestinal factor is further modified as a result of changes in the intestinal flora and variations in the destruction of bile acids by bacterial action. The functional state of the hepatic cells must also be taken into consideration, for changes in the functional efficiency of the liver as a result of the production of an Eck fistula, cholangitis, or the administration of hepatic toxins, such as chloroform or phosphorus have a profound influence on the excretion of the bile acids. Greene and Snell (1928) found the variation in the attainable concentration of bile acids in the bile to be relatively slight, at least when compared to the observed variations in the concentration of bilirubin. Under some conditions, therefore, the volume of the biliary output may be determined by the excretion of the bile acids, although this relation does not always hold and the two may be entirely independent the one of the other.

Direct evidence regarding the secretion of bile in normal men, and the modifications accompanying disease, is lacking. The greater part of the available data is concerned with the composition of bile from the gallbladder but because of the concentrating action of that viscus such analyses do not indicate the composition of the bile as initially formed in the liver. Similar criticisms apply to the studies of Rosenthal and von Falkenhausen (1923), and of McClure (1925) and his associates, who used the material obtained by duodenal drainage. Apart from the uncertainty, as to how far the duodenal content is representative of the hepatic secretion, there is further difficulty in that only the concentration, and not the total amount of the different biliary constituents, is determinable. Numerous studies have been made of bile obtained from patients with permanent biliary fistulas. Unfortunately, the majority of such investigations was made before 
the development of adequate analytic methods and so were concerned chiefly with the changes in the volume and total solids present in the bile.

More recently von Czyhlarz, Fuchs and von Fürth (1913), Chabrol, Bénard and Bariéty (1926), and Rosenthal, von Falkenhausen and Freund (1926) have reported single cases or small groups of cases in which the composition of the bile has been carefully studied during the first few days after cholecystectomy and drainage of the common bile duct. We wish to report the changes in an additional series of nine cases. Such cases afford an opportunity to study the effect of biliary obstruction on the secretion of bile and the return toward normal following relief of the obstruction. It is possible, in consequence, to compare the changes observed clinically in patients with those in dogs reported by McMaster, Broun and Rous (1923) following temporary occlusion of a permanent biliary fistula. Furthermore, the differences in the response of the different cases afford additional light on the disturbances in the functional activity of the liver as a result of biliary obstruction or infection.

\section{DETAILS OF INVESTIGATION}

The drainage of bile from the gallbladder or common bile duct, subsequent to the relief of biliary obstruction, was studied in nine cases. Details regarding the clinical histories of certain of these cases are given elsewhere (26). The methods-of analysis were those used in previous studies of this series: the method of Greene, Snell and Walters (1925) for bilirubin; of Aldrich and Bledsoe (1928) for bile acids; of Van Slyke (10) for chlorides, and of Van Slyke and Cullen (10) for urea. The data obtained in the different cases are given in the accompanying tables. It is recognized that the collection of bile from a drainage tube inserted in the gallbladder or common bile duct is not always complete. In the present series the analyis of bile was continued during the whole period of observation but the tables include only the data obtained during the period when the collection was apparently complete. For purposes of study the cases have been divided into three groups.

Group 1. Tables 1, 2 and 3, and figure $1 A$ comprise three cases of chronic cholecystitis with stone in the common bile duct. In these 
cases, the histories were of typical, intermittent biliary obstruction, with mild degrees of jaundice. The liver was apparently normal at the time of operation. Cholecystectomy, choledocholithotomy and choledochostomy were performed in all, and the postoperative course was uneventful.

Group 2. Tables 4,5 and 6 and figure $1 B$ contain three cases. One patient had chronic cholangitis, biliary obstruction and a consequent obstructive type of biliary cirrhosis; a T-tube was inserted in the common bile duct. Another patient had chronic cholecystitis with stones, a stone in the common bile duct, and considerable biliary cirrhosis; a T-tube was inserted in the common bile duct after removal of the stone. The third patient had complete biliary obstruction due to carcinoma of the head of the pancreas, but also had cirrhotic changes in the liver in consequence of the obstruction; cholecystostomy, as a preliminary to cholecystenterostomy, was performed.

Group 3. Increase in the biliary drainage, with paling and thinning of the bile, is one of the symptoms of postoperative hepatic insuffciency in cases of obstructive jaundice. Walters and Parham (1922) have pointed out the serious prognostic importance of cholerrhagia. This condition was present in the three cases in group 3 (tables 7,8 and 9 and figures $1 C$ and $D$ ). The patients gave a history of marked and painless jaundice of from two to six months' duration. In two cases there was a carcinoma of the head of the pancreas and cholecystostomy was done, preliminary to cholecystenterostomy. In the third patient there was a carcinoma of the common and hepatic bile ducts, and only choledochostomy could be done.

\section{COMMENT}

McMaster, Broun and Rous (1923) have described in detail the changes, in dogs, produced in the character of the bile as a result of obstruction to a permanent biliary fistula and the response to the relief of such an obstruction. They pointed out that with partial biliary obstruction there was a reduction in the output of pigments, of bile acids and of cholesterol, and to a much greater degree than that in the volume of bile, so that the fluid elaborated by the liver became progressively poorer in the typical biliary constituents. Because of the analogy with changes in the renal secretion incident to the develop- 


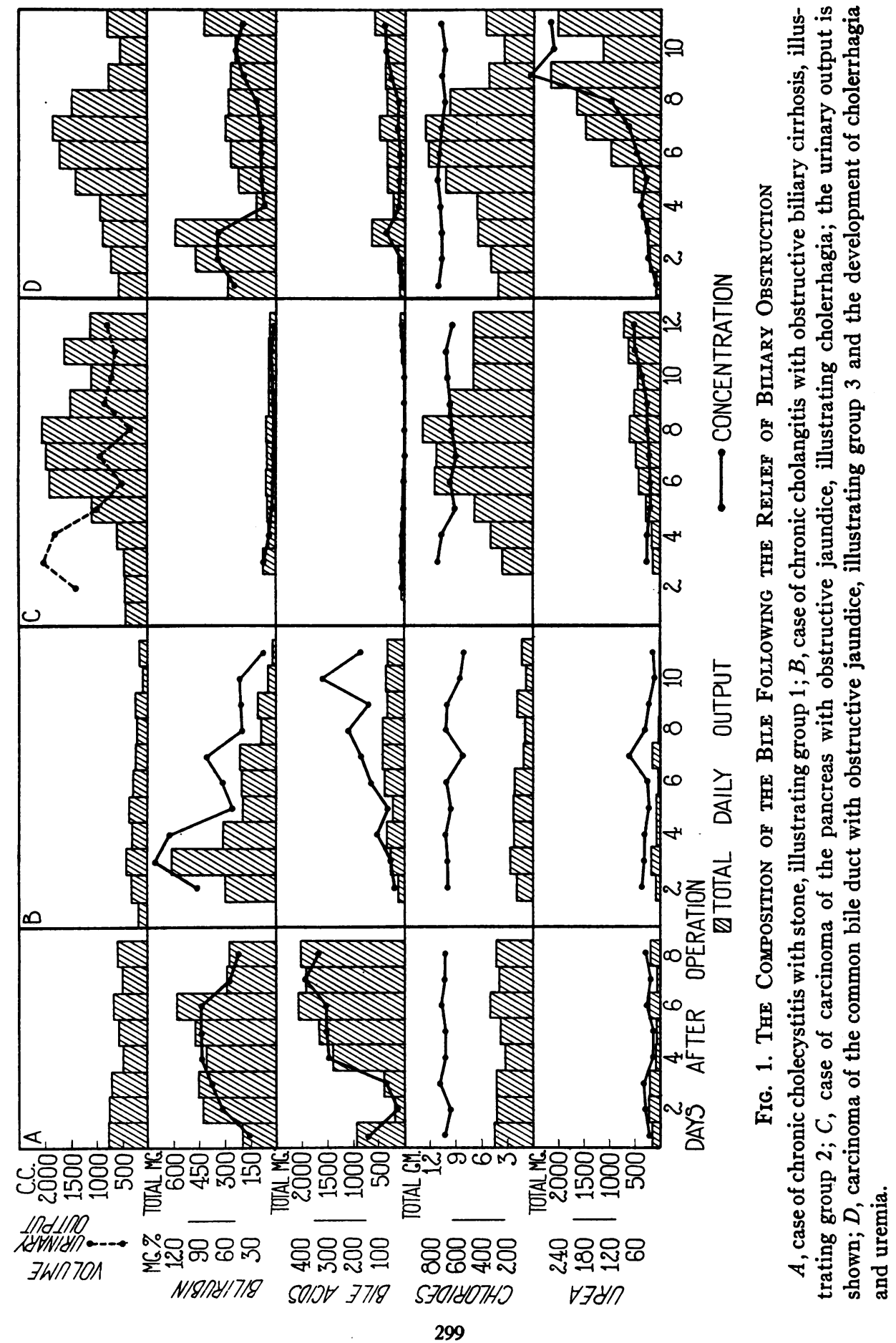


ment of hydronephrosis, they used the term hydrohepatosis to designate the changes in the liver. They also pointed out that in the presence of complete biliary obstruction, the concentrating activity of the gallbladder may mask these changes either in whole or in part. Following relief of the biliary obstruction, a copious amount of bile was elaborated by the liver and the flow persisted until the greater part of the retained biliary constitunts had been eliminated. This bile was more dilute than normal although the increase in volume was

TABLE 1

Summary of data in case 1 (group 1)

\begin{tabular}{|c|c|c|c|c|c|c|c|c|c|c|c|c|}
\hline \multirow{3}{*}{$\begin{array}{l}\text { Days } \\
\text { after } \\
\text { opera- } \\
\text { tion }\end{array}$} & \multicolumn{9}{|c|}{ Bile } & \multirow{2}{*}{\multicolumn{3}{|c|}{ Blond serum* }} \\
\hline & \multirow{2}{*}{$\begin{array}{l}\text { Vol- } \\
\text { ume }\end{array}$} & \multicolumn{2}{|c|}{ Bilirubin } & \multicolumn{2}{|c|}{$\begin{array}{c}\text { Bile acids as } \\
\text { glycocholic acid }\end{array}$} & \multicolumn{2}{|c|}{ Urea } & \multicolumn{2}{|c|}{$\begin{array}{c}\text { Chlorides as } \\
\text { sodium chloride }\end{array}$} & & & \\
\hline & & $\begin{array}{l}\text { Concen- } \\
\text { tration }\end{array}$ & Total & $\begin{array}{l}\text { Concen- } \\
\text { tration }\end{array}$ & Total & $\begin{array}{l}\text { Concen- } \\
\text { tration }\end{array}$ & Total & $\begin{array}{l}\text { Concen- } \\
\text { tration }\end{array}$ & Total & $\begin{array}{l}\text { Bili- } \\
\text { rubin }\end{array}$ & Urea & $\begin{array}{l}\text { Sodium } \\
\text { chloride }\end{array}$ \\
\hline & $c c$. & $\begin{array}{c}\text { mgm. } \\
\text { per } 100 \\
\text { cc. }\end{array}$ & $m g m$. & $\begin{array}{c}\text { mgm. } \\
\text { per } 100 \\
c c .\end{array}$ & $m g m$. & $\begin{array}{c}\text { mgm. } \\
\text { per } 100 \\
\text { ce. }\end{array}$ & mgm. & $\begin{array}{c}\text { mgm. } \\
\text { per } 100 \\
\text { cc. }\end{array}$ & grams & $\begin{array}{c}\text { mgm. } \\
\text { per } 100 \\
\text { cc. }\end{array}$ & $\begin{array}{c}\text { mgm. } \\
\text { per } 100 \\
\text { cc. }\end{array}$ & $\begin{array}{c}\text { per } 100 \\
\text { cc. }\end{array}$ \\
\hline 1 & 650 & 31 & 200 & 145 & 940 & 25 & 162 & 693 & 4.50 & & & \\
\hline 2 & 655 & 64 & 425 & 26 & 170 & 36 & 236 & 645 & 4.23 & & & \\
\hline 3 & 600 & 75 & 450 & 70 & 420 & 39 & 234 & 723 & 4.35 & & & \\
\hline 4 & 475 & 87 & 414 & 296 & 1,410 & 18 & 85 & 681 & 3.24 & & 38 & 585 \\
\hline 5 & 550 & 87 & 478 & 308 & $\mid 1,690$ & 18 & 99 & 681 & 3.75 & & & \\
\hline 6 & 675 & 87 & 587 & 308 & 2,080 & 32 & 216 & 708 & 4.77 & & & \\
\hline 7 & 500 & 56 & 280 & 387 & 1,930 & 24 & 120 & 692 & 3.46 & & & \\
\hline 8 & 600 & 45 & 270 & 339 & 2,040 & 36 & 216 & 687 & 4.13 & & & \\
\hline
\end{tabular}

* Before operation the following values in milligrams in each $100 \mathrm{cc}$. were determined: serum bilirubin, 2.8; blood urea, 24; serum chlorides as sodium chloride, 613 .

such that the total output of pigment was increased during the period of choleresis.

Similar changes are found in many cases following the relief of biliary obstruction. The initial changes depend on the character of the bile present in the biliary tract at the time of operation. If it is thick and viscid as a result of the concentrating action of the gallbladder, there is an initial decrease in the concentration of the biliary drainage as this retained "stasis" bile is washed out (tables 1 and 2). If, on the other hand, "white" bile is present in the bile ducts, the fluid initially obtained may contain only minimal quantities of bili- 
C. H. GREENE, W. WALTERS AND C. H. FREDRICKSON

TABLE 2

Summary of data in case 2 (group 1)

\begin{tabular}{|c|c|c|c|c|c|c|c|c|c|c|c|c|}
\hline \multirow{3}{*}{$\begin{array}{l}\text { Days } \\
\text { after } \\
\text { opera- } \\
\text { tion }\end{array}$} & \multicolumn{9}{|c|}{ Bile } & \multirow{2}{*}{\multicolumn{3}{|c|}{ Blood serum* }} \\
\hline & \multirow{2}{*}{$\begin{array}{l}\text { Vol- } \\
\text { ume }\end{array}$} & \multicolumn{2}{|c|}{ Bilirubin } & \multicolumn{2}{|c|}{$\begin{array}{c}\text { Bile acids as } \\
\text { glycocholic acid }\end{array}$} & \multicolumn{2}{|c|}{ Urea } & \multicolumn{2}{|c|}{$\begin{array}{c}\text { Chlorides as } \\
\text { sodium chloride }\end{array}$} & & & \\
\hline & & $\begin{array}{l}\text { Concen- } \\
\text { tration }\end{array}$ & Total & $\begin{array}{l}\text { Concen- } \\
\text { tration }\end{array}$ & Total & $\begin{array}{l}\text { Concen- } \\
\text { tration }\end{array}$ & Total & $\begin{array}{l}\text { Concen- } \\
\text { tration }\end{array}$ & Total & $\begin{array}{l}\text { Bili- } \\
\text { rubin }\end{array}$ & Urea & $\begin{array}{l}\text { Sodium } \\
\text { chloride }\end{array}$ \\
\hline & $c c$. & $\begin{array}{c}\text { mgm. } \\
\text { per } 100 \\
c c .\end{array}$ & $m g m$. & $\begin{array}{c}\text { mggm. } \\
\text { per } 100 \\
\text { cc. }\end{array}$ & $m g m$. & $\begin{array}{c}\underset{\text { per } 100}{\text { mgm. }} \\
\text { cc. }\end{array}$ & $m g m$. & $\begin{array}{c}\text { per } 100 \\
\text { cc. }\end{array}$ & grams & $\begin{array}{c}\text { per } 100 \\
\text { cc. }\end{array}$ & $\begin{array}{c}\text { mgm. } \\
\text { per } 100 \\
\text { cc. }\end{array}$ & $\begin{array}{c}\underset{\text { per } 100}{\text { cc. }} \\
\text { cc. }\end{array}$ \\
\hline 1 & 130 & & & & & & & & & & & \\
\hline 2 & 190 & 128 & 244 & 138 & 262 & 50 & 95 & 689 & 1.31 & & & \\
\hline 3 & 250 & 97 & 243 & 134 & 335 & 37 & 92 & 617 & 1.54 & 1.3 & 25 & 511 \\
\hline 4 & 175 & 71 & 124 & 333 & 583 & 24 & 41 & 675 & 1.18 & & & \\
\hline 5 & 215 & 51 & 110 & 397 & 855 & 20 & 43 & 675 & 1.45 & & & \\
\hline 6 & 200 & 77 & 154 & 513 & 1,026 & 13 & 26 & 648 & 1.29 & & & \\
\hline 7 & 175 & 101 & 177 & 545 & 955 & 21 & 37 & 611 & 1.07 & & & \\
\hline 8 & 150 & 112 & 168 & 450 & 675 & 16 & 24 & 619 & 0.93 & & & \\
\hline 9 & 150 & 103 & 155 & 800 & 1,200 & 13 & 20 & 628 & 0.94 & & 24 & 549 \\
\hline 10 & 145 & 62 & 90 & 1,064 & 1,540 & 8 & 12 & 539 & 0.78 & & & \\
\hline
\end{tabular}

* Before operation the following values in milligrams in each $100 \mathrm{cc}$. were determined: serum bilirubin, 1.5; blood urea, 25; serum chlorides as sodium chloride, 519 .

TABLE 3

Summary of data in case 3 (group 1)

\begin{tabular}{|c|c|c|c|c|c|c|c|c|c|c|c|c|}
\hline \multirow{3}{*}{$\begin{array}{l}\text { Days } \\
\text { after } \\
\text { opera- } \\
\text { tion }\end{array}$} & \multicolumn{9}{|c|}{ Bile } & \multirow{2}{*}{\multicolumn{3}{|c|}{ Blood serum* }} \\
\hline & \multirow{2}{*}{$\begin{array}{l}\text { Vol- } \\
\text { ume }\end{array}$} & \multicolumn{2}{|c|}{ Bilirubin } & \multicolumn{2}{|c|}{$\begin{array}{c}\text { Bile acids as } \\
\text { glycocholic acid }\end{array}$} & \multicolumn{2}{|c|}{ Urea } & \multicolumn{2}{|c|}{$\begin{array}{c}\text { Chlorides as } \\
\text { sodium chloride }\end{array}$} & & & \\
\hline & & $\begin{array}{l}\text { Concen- }- \\
\text { tration }\end{array}$ & Total & $\begin{array}{l}\text { Concen- }- \\
\text { tration }\end{array}$ & Total & $\begin{array}{l}\text { Concen- } \\
\text { tration }\end{array}$ & Total & $\begin{array}{l}\text { Concen- } \\
\text { tration }\end{array}$ & Total & $\begin{array}{l}\text { Bili- } \\
\text { rubin }\end{array}$ & Urea & $\begin{array}{l}\text { Sodium } \\
\text { chloride }\end{array}$ \\
\hline & $c c$. & $\begin{array}{c}\underset{\text { per } 100}{\operatorname{mgm}} \\
\text { cc. }\end{array}$ & $m g m$. & $\begin{array}{c}\text { mgm. } \\
\text { per } 100 \\
\text { cc. }\end{array}$ & mgm. & $\begin{array}{c}\underset{\text { per } 100}{\operatorname{mgm}} \\
\text { cc. }\end{array}$ & mgm. & $\begin{array}{c}\text { mgm. } \\
\text { per } 100 \\
\text { cc. }\end{array}$ & grams & $\begin{array}{c}\operatorname{mgm} . \\
\text { per } 100 \\
\text { cc. }\end{array}$ & $\begin{array}{c}\text { mgm. } \\
\text { per } 100 \\
c c .\end{array}$ & $\begin{array}{c}\text { mgm. } \\
\text { per } 100 \\
\text { cc. }\end{array}$ \\
\hline 1 & 200 & & & & & & & & & & & \\
\hline 2 & 350 & & & & & & & & & & & \\
\hline 3 & 365 & 44 & 160 & 66 & 240 & 50 & 183 & 619 & 2.26 & & & \\
\hline 4 & 700 & 19 & 133 & 62 & 434 & 55 & 385 & 460 & 3.32 & 3.6 & 24 & 507 \\
\hline 5 & 600 & 79 & 474 & 387 & 2,320 & 92 & 550 & 723 & 4.34 & & & \\
\hline
\end{tabular}

* Before operation the following values in milligrams in each $100 \mathrm{cc}$. were determined: serum bilirubin, 3.8; blood urea, 15; serum chlorides as sodium chloride, 1 .

rubin or bile acids. The changes reported by Rosenthal, von Falkenhausen and Freund (1926), and by Chabrol, Bénard and Bariéty (1926) 
TABLE 4

Summary of data in case 4 (group 2)

\begin{tabular}{|c|c|c|c|c|c|c|c|c|c|c|c|c|}
\hline \multirow{3}{*}{$\begin{array}{c}\text { Days } \\
\text { atter } \\
\text { opera- } \\
\text { tion }\end{array}$} & \multicolumn{9}{|c|}{ Bile } & \multirow{2}{*}{\multicolumn{3}{|c|}{ Blood serum* }} \\
\hline & \multirow{2}{*}{$\begin{array}{l}\text { Vol- } \\
\text { ume }\end{array}$} & \multicolumn{2}{|c|}{ Bilirubin } & \multicolumn{2}{|c|}{$\begin{array}{c}\text { Bile acids as } \\
\text { glycocholic acid }\end{array}$} & \multicolumn{2}{|c|}{ Urea } & \multicolumn{2}{|c|}{$\begin{array}{l}\text { Chlorides as } \\
\text { sodium chloride }\end{array}$} & & & \\
\hline & & $\begin{array}{l}\text { Concen- }- \\
\text { tration }\end{array}$ & Total & $\begin{array}{l}\text { Concen- } \\
\text { tration }\end{array}$ & Total & $\begin{array}{l}\text { Concen- }- \\
\text { tration }\end{array}$ & Total & $\begin{array}{l}\text { Concen- }- \\
\text { tration }\end{array}$ & Total & $\begin{array}{l}\text { Bili- } \\
\text { rubin }\end{array}$ & Urea & $\begin{array}{l}\text { Sodium } \\
\text { chloride }\end{array}$ \\
\hline & $c c$. & $\begin{array}{l}\text { mgm. } \\
\text { per } 100 \\
\text { cc. }\end{array}$ & $m g m$. & $\begin{array}{l}\text { mgm. } \\
\text { per } 100 \\
\text { cc. }\end{array}$ & $m g m$. & $\begin{array}{c}\underset{\text { mgm. }}{\text { per } 100} \\
\text { cc. }\end{array}$ & $m g m$. & $\begin{array}{l}\underset{\text { pgm. }}{\text { per } 100} \\
\text { cc. }\end{array}$ & $m g m$. & $\begin{array}{l}\underset{\text { per }}{\text { mog. }} \text {.0 } \\
\text { cc. }\end{array}$ & $\begin{array}{l}\text { mgm. } \\
\text { per } 100 \\
\text { cc. }\end{array}$ & $\begin{array}{l}\text { pgm. } \\
\text { per } 100 \\
\text { cc. }\end{array}$ \\
\hline 1 & 200 & & & & & & & & & & & \\
\hline 2 & 320 & 94 & 300 & 40 & 128 & 51 & 163 & 663 & 2.12 & & & \\
\hline 3 & 425 & 143 & 607 & 60 & 255 & 47 & 200 & 673 & 2.86 & & & \\
\hline 4 & 325 & 98 & 319 & 106 & 345 & 46 & 150 & 691 & 2.25 & 3.7 & 36 & 519 \\
\hline 5 & 365 & 51 & 186 & 68 & 248 & 30 & 110 & 645 & 2.35 & & & \\
\hline 6 & 290 & 68 & 197 & 137 & 400 & 35 & 101 & 677 & 1.96 & & & \\
\hline 7 & 250 & 83 & 208 & 174 & 435 & 78 & 195 & 575 & 1.44 & & & \\
\hline 8 & 200 & 42 & 82 & 222 & 444 & 40 & 80 & 688 & 1.37 & & & \\
\hline 9 & 250 & 46 & 115 & 141 & 353 & 30 & 75 & 680 & 1.70 & & & \\
\hline 10 & 120 & 49 & 59 & 321 & 386 & 17 & 20 & 572 & 0.69 & & & \\
\hline 11 & 200 & 16 & 32 & 173 & 346 & 20 & 40 & 541 & 1.08 & & & \\
\hline
\end{tabular}

* Before operation the following values in milligrams in each $100 \mathrm{cc}$. were determined: serum bilirubin, 2.1; blood urea, 24 .

TABLE 5

Summary of data in case 5 (group 2)

\begin{tabular}{|c|c|c|c|c|c|c|c|c|c|c|c|c|}
\hline \multirow{3}{*}{$\begin{array}{l}\text { Days } \\
\text { after } \\
\text { opera- } \\
\text { tion }\end{array}$} & \multicolumn{9}{|c|}{ Bile } & \multirow{2}{*}{\multicolumn{3}{|c|}{ Blood serum* }} \\
\hline & \multirow{2}{*}{$\begin{array}{l}\text { Vol- } \\
\text { ume }\end{array}$} & \multicolumn{2}{|c|}{ Bilirubin } & \multicolumn{2}{|c|}{$\begin{array}{c}\text { Bile acids as } \\
\text { glycocholic acid }\end{array}$} & \multicolumn{2}{|c|}{ Urea } & \multicolumn{2}{|c|}{$\mid \begin{array}{c}\text { Chlorides as } \\
\text { sodium chloride }\end{array}$} & & & \\
\hline & & $\begin{array}{l}\text { Concen- } \\
\text { tration }\end{array}$ & Total & $\begin{array}{c}\text { Concen- } \\
\text { tration }\end{array}$ & Total & $\begin{array}{l}\text { Concen- } \\
\text { tration }\end{array}$ & Total & $\begin{array}{l}\text { Concen- } \\
\text { tration }\end{array}$ & Total & $\begin{array}{l}\text { Bili- } \\
\text { rubin }\end{array}$ & Urea & $\begin{array}{l}\text { Sodium } \\
\text { chloride }\end{array}$ \\
\hline & $c c$ & $\begin{array}{c}\text { mgm. } \\
\text { per } 100 \\
\text { cc. }\end{array}$ & $m g m$. & $\begin{array}{c}\underset{\text { per } 100}{\text { mgm. }} \\
\text { cc. }\end{array}$ & $m g m$. & $\begin{array}{c}\underset{\text { per } 100}{\operatorname{mgm}} \\
\text { cc. }\end{array}$ & $m g m$. & $\begin{array}{c}\text { mgm. } \\
\text { per } 100 \\
\text { cc. }\end{array}$ & $\boldsymbol{m g} \boldsymbol{m}$ & $\begin{array}{c}\text { mgm. } \\
\text { per } 100 \\
\text { cc. }\end{array}$ & $\begin{array}{c}\text { mgm. } \\
\text { per } 100 \\
\text { cc. }\end{array}$ & $\begin{array}{c}\text { mgm. } \\
\text { per } 100 \\
\text { cc. }\end{array}$ \\
\hline 1 & 450 & & & & & & & & & & & \\
\hline 2 & 300 & 56 & 168 & 13 & 39 & 49 & 147 & 705 & 2.11 & & & \\
\hline 3 & 425 & 34 & 145 & 29 & 123 & 51 & 217 & 843 & 3.58 & 5.6 & 48 & 551 \\
\hline 4 & 350 & 57 & 200 & 71 & 248 & 26 & 87 & 723 & 2.53 & & & \\
\hline 5 & 400 & 8 & 32 & 111 & 444 & 29 & 116 & 739 & 2.95 & & & \\
\hline 6 & 615 & 11 & 68 & 45 & 277 & 20 & 123 & 600 & 3.69 & & & \\
\hline 7 & 390 & 37 & 144 & 119 & 465 & 17 & 66 & 711 & 2.77 & & & \\
\hline 8 & 320 & 12 & 38 & 88 & 281 & 35 & 112 & 751 & 2.40 & & & \\
\hline 9 & 200 & 20 & 40 & & & 49 & 98 & 789 & 1.58 & & & \\
\hline 10 & 325 & 22 & 71 & 112 & 364 & 27 & 88 & 702 & 2.28 & & 42 & 592 \\
\hline
\end{tabular}

* Before operation the following values in milligrams in each $100 \mathrm{cc}$. were determined: serum bilirubin, 5.6 ; blood urea, 26. 
were similar to those observed in group 1 in this series. In all (tables 1,2 and 3 , and figure $1 A$ ), there was a secretion of a moderate amount of bile of good color. There was a rapid increase in both the concentration and total output of bilirubin during the greater part of the first week with a subsequent decrease to the apparent normal level. This initial increase may be interpreted as evidence of a washing out of retained bile pigment, although it is recognized that some of the

TABLE 6

Summary of data in case 6 (group 2)

\begin{tabular}{|c|c|c|c|c|c|c|c|c|c|c|c|c|}
\hline \multirow{3}{*}{$\begin{array}{c}\text { Days } \\
\text { after } \\
\text { opera- } \\
\text { tion }\end{array}$} & \multicolumn{9}{|c|}{ Bile } & \multirow{2}{*}{\multicolumn{3}{|c|}{ Blood serum* }} \\
\hline & \multirow{2}{*}{$\begin{array}{l}\text { Vol- } \\
\text { ume }\end{array}$} & \multicolumn{2}{|c|}{ Bilirubin } & \multicolumn{2}{|c|}{$\begin{array}{c}\text { Bile acids as } \\
\text { glycocholic acid }\end{array}$} & \multicolumn{2}{|c|}{ Urea } & \multicolumn{2}{|c|}{$\begin{array}{c}\text { Chlorides as } \\
\text { sodium chloride }\end{array}$} & & & \\
\hline & & $\begin{array}{c}\text { Concen- } \\
\text { tration }\end{array}$ & Total & $\begin{array}{c}\text { Concen- } \\
\text { tration }\end{array}$ & Total & $\begin{array}{l}\text { Concen- } \\
\text { tration }\end{array}$ & Total & $\begin{array}{l}\text { Concen- } \\
\text { tration }\end{array}$ & Total & $\begin{array}{c}\text { Bili- } \\
\text { rubin }\end{array}$ & Urea & $\begin{array}{l}\text { Sodium } \\
\text { chloride }\end{array}$ \\
\hline & $c c$. & $\begin{array}{c}\text { mgm. } \\
\text { per } 100 \\
c c .\end{array}$ & $m g m$. & $\begin{array}{c}\underset{\text { per } 100}{\text { cc. }} \\
\text { cc. }\end{array}$ & $m g m$. & $\begin{array}{c}\underset{\text { per } 100}{ } \\
c c .\end{array}$ & mgm. & $\begin{array}{c}\text { mgm. } \\
\text { per } 100 \\
c c .\end{array}$ & $m g m$. & $\begin{array}{c}\text { mgm. } \\
\text { per } 100 \\
\text { cc. }\end{array}$ & $\begin{array}{c}\underset{\text { per } 100}{\text { mgm. }} \\
\text { cc. }\end{array}$ & $\begin{array}{c}\underset{\text { pgm }}{\text { mer }} 100 \\
\text { cc. }\end{array}$ \\
\hline 1 & 250 & & & & & & & & & & & \\
\hline 2 & 300 & 14 & 42 & 58 & 174 & 29 & 87 & 767 & 2.3 & & & \\
\hline 3 & 325 & 20 & 65 & 57 & 185 & 15 & 50 & 767 & 2.5 & 11.0 & 19 & \\
\hline 4 & 250 & 20 & 50 & 40 & 100 & 7 & 18 & 797 & 2.0 & & & \\
\hline 5 & 270 & 29 & 80 & 36 & 96 & 15 & 40 & 787 & 2.1 & 10.0 & 19 & 559 \\
\hline 6 & 300 & 6 & 78 & 29 & 90 & 14 & 42 & 781 & 2.3 & & & \\
\hline 7 & 300 & 22 & 66 & 32 & 93 & 17 & 51 & 725 & 2.2 & & & \\
\hline 8 & 270 & 17 & 47 & 36 & 98 & 14 & 38 & 741 & 2.0 & & & \\
\hline 9 & 240 & 17 & 40 & 33 & 79 & 15 & 36 & 743 & 1.8 & & & \\
\hline 10 & 280 & 13 & 35 & 37 & 103 & 19 & 53 & 760 & 2.1 & 8.2 & & \\
\hline 11 & 350 & 20 & 50 & 36 & 117 & 19 & 66 & 720 & 1.8 & & & \\
\hline 12 & 300 & 29 & 89 & & & 28 & 81 & 761 & 2.2 & & & \\
\hline
\end{tabular}

* Before operation the following values in milligrams in each $100 \mathrm{cc}$. were determined: serum bilirubin, 16.2.

increase in bilirubin may be explained by hemorrhage and trauma at the time of operation. Both the concentration and the total output of bile salts were reduced in the bile first formed after the relief of the obstruction. Recovery was rapid, however, and within a week the concentration in the bile compared favorably with that seen in hepatic bile from normal dogs (tables 1, 2 and 3 , and figure $1 A$ ). This concentration and the total output of bile would seem to be well maintained thereafter. These changes would seem to be typical of the 
response when the obstruction to the biliary passages had not been sufficiently long continued or complete to produce permanent hepatic injury. Smyth and Whipple (1924) pointed out that in dogs a reduction in the excretion of bile acids is produced by a dose of chloroform so small as to be incapable of causing any recognizable structural change in the hepatic epithelium. Similarly, reduction or cessation in the production of bile acids would seem to be a characteristic effect

TABLE 7

Summary of data in case 7 (group 3)

\begin{tabular}{|c|c|c|c|c|c|c|c|c|c|c|c|c|c|}
\hline \multirow{3}{*}{$\begin{array}{c}\text { Days } \\
\text { after } \\
\text { opera- } \\
\text { tion }\end{array}$} & \multirow{3}{*}{$\begin{array}{l}\text { Urine in } \\
\text { twenty- } \\
\text { four } \\
\text { hours }\end{array}$} & \multicolumn{9}{|c|}{ Bile } & \multirow{2}{*}{\multicolumn{3}{|c|}{ Blood serum* }} \\
\hline & & \multirow{2}{*}{ Volume } & \multicolumn{2}{|c|}{ Bilirubin } & \multicolumn{2}{|c|}{$\begin{array}{c}\text { Bile acids as } \\
\text { glycocholic } \\
\text { acid }\end{array}$} & \multicolumn{2}{|c|}{ Urea } & \multicolumn{2}{|c|}{$\begin{array}{l}\text { Chlorides as } \\
\text { sodium chlo- } \\
\text { ride }\end{array}$} & & & \\
\hline & & & $\begin{array}{c}\text { Con- } \\
\text { centra- } \\
\text { tion }\end{array}$ & Total & $\begin{array}{c}\text { Con- } \\
\text { centra- } \\
\text { tion }\end{array}$ & Total & $\begin{array}{c}\text { Con- } \\
\text { centra- } \\
\text { tion }\end{array}$ & Total & $\begin{array}{c}\text { Con- } \\
\text { centra- } \\
\text { tion }\end{array}$ & Total & $\begin{array}{c}\text { Bili- } \\
\text { rubin }\end{array}$ & Urea & $\begin{array}{l}\text { So- } \\
\text { dium } \\
\text { chlo- } \\
\text { ride }\end{array}$ \\
\hline & $c c$. & $c c$. & $\begin{array}{c}\text { mgm. } \\
\text { per } 100 \\
c c .\end{array}$ & $m g m$. & $\begin{array}{c}\text { mgm. } \\
\text { per } 100 \\
c c .\end{array}$ & $m g m$. & $\underset{\substack{\text { per } 100 \\
c c .}}{\operatorname{mgm}}$ & $m g m$. & $\begin{array}{c}\operatorname{mgm} . \\
\text { per } 100 \\
c c .\end{array}$ & mgm. & $\begin{array}{c}\text { mgmm. } \\
\text { per } 100 \\
c c .\end{array}$ & $\begin{array}{c}\text { mgmm. } \\
\text { per } 100 \\
c c .\end{array}$ & $\begin{array}{l}\text { mgm. } \\
\text { per } 100 \\
c c .\end{array}$ \\
\hline 1 & & 450 & & & & & & & & & & & \\
\hline 2 & 1,425 & 475 & & & 13 & 62 & & & & & & & \\
\hline 3 & 2,025 & 480 & 16 & 77 & 17 & 82 & 35 & 168 & 752 & 3.62 & 16.5 & 41 & \\
\hline 4 & 1,800 & 650 & 9 & 58 & 11 & 71 & 35 & 227 & 711 & 4.62 & & & 519 \\
\hline 5 & 1,000 & 1,100 & 5 & 55 & 4 & 44 & 25 & 275 & 609 & 6.70 & & & \\
\hline 6 & 500 & 1,825 & 4 & 73 & 3 & 55 & 22 & 402 & 649 & 11.84 & & & \\
\hline 7 & 950 & 1,950 & 3 & 59 & 1 & 19 & 26 & 506 & 601 & 11.70 & 13.5 & & \\
\hline 8 & 350 & 2,050 & 3 & 61 & 2 & 41 & 31 & 635 & 637 & 13.05 & & & \\
\hline 9 & 850 & 1,550 & 3 & 46 & 1 & 16 & 33 & 512 & 641 & 9.95 & & & \\
\hline 10 & 700 & 1,100 & 3 & 33 & 1 & 11 & 45 & 495 & 667 & 7.34 & 13.7 & 33 & \\
\hline 11 & 650 & 1,650 & 4 & 46 & 6 & 69 & 60 & 690 & 685 & 7.87 & & & \\
\hline 12 & 800 & 1,240 & 3 & 37 & 7 & 87 & 63 & 781 & 633 & 7.85 & 14.3 & 38 & 639 \\
\hline
\end{tabular}

* Before operation the following values were determined: urine, cc. in 24 hours, 606; serum bilirubin, mgm. in each 100 cc., 25; serum urea, mgm. in each 100 cc., 15.

of biliary obstruction, and confirmation of this view is furnished by the experiments of Brakefield and Schmidt (1926) and of Snell, Greene and Rowntree (1927). If the injury to the liver in consequence of the obstruction is not too great, recovery is rapid and the concentration and total amount of bile acids in the bile rapidly return toward normal. In no case was there evidence for the washing out of any appreciable quantity of retained bile acids. 
Hepatic injury and the development of obstructive biliary cirrhosis, as in the cases in group 2, modified the response of the liver to the relief of the obstruction (tables 4,5 and 6 , and figure $1 B$ ). The changes in the curve of excretion of bilirubin were not significantly different from those in the preceding group of cases although possibly the values were reduced slightly. Both the concentration and the total output of bile acids, on the other hand, were reduced and remained low throughout the period of observation. We believe this

TABLE 8

Summary of data in case 8 (group 3)

\begin{tabular}{|c|c|c|c|c|c|c|c|c|c|c|c|c|}
\hline \multirow{3}{*}{$\begin{array}{l}\text { Days } \\
\text { after } \\
\text { opera- } \\
\text { tion }\end{array}$} & \multicolumn{9}{|c|}{ Bile } & \multirow{2}{*}{\multicolumn{3}{|c|}{ Blood serum* }} \\
\hline & \multirow{2}{*}{$\begin{array}{l}\text { Vol- } \\
\text { ume }\end{array}$} & \multicolumn{2}{|c|}{ Bilirubin } & \multicolumn{2}{|c|}{$\begin{array}{c}\text { Bile acids as } \\
\text { glycocholic acid }\end{array}$} & \multicolumn{2}{|c|}{ Urea } & \multicolumn{2}{|c|}{$\begin{array}{c}\text { Chlorides as } \\
\text { sodium chloride }\end{array}$} & & & \\
\hline & & $\begin{array}{l}\text { Concen- } \\
\text { tration }\end{array}$ & Total & $\begin{array}{l}\text { Concen- } \\
\text { tration }\end{array}$ & Total & $\begin{array}{l}\text { Concen- } \\
\text { tration }\end{array}$ & Total & $\begin{array}{l}\text { Concen- } \\
\text { tration }\end{array}$ & Total & $\begin{array}{l}\text { Bili- } \\
\text { rubin }\end{array}$ & Urea & $\begin{array}{l}\text { Sodium } \\
\text { chloride }\end{array}$ \\
\hline & $c c$. & $\begin{array}{c}\text { per } 100 \\
\text { cc. }\end{array}$ & $m g m$. & $\begin{array}{c}\text { mgm. } \\
\text { per } 100 \\
c c .\end{array}$ & mgm. & $\begin{array}{c}\text { mgm. } \\
\text { per } 100 \\
\text { cc. }\end{array}$ & mgm. & $\begin{array}{c}\text { mgm. } \\
\text { per } 100 \\
\text { cc. }\end{array}$ & $m g m$. & $\begin{array}{c}\underset{\text { per } 100}{\operatorname{mgm}} \\
\text { cc. }\end{array}$ & $\begin{array}{c}\underset{\text { per } 100}{ } . \\
\text { cc. }\end{array}$ & $\begin{array}{c}\text { mgm. } \\
\text { per } 100 \\
\text { cc. }\end{array}$ \\
\hline $\begin{array}{l}1 \\
2\end{array}$ & $\begin{array}{l}1,935 \\
3,025\end{array}$ & & & & & & & & & & & \\
\hline 3 & 1,635 & 9 & 155 & & & 21 & 330 & & & 7.5 & 28 & \\
\hline 4 & 1,540 & 10 & 155 & & & 21 & 320 & & & & & \\
\hline 5 & 1,530 & 10 & 153 & 15 & 237 & 17 & 260 & 540 & 8.31 & 7.2 & 17 & 565 \\
\hline 6 & 1,750 & 9 & 161 & 16 & 284 & 17 & 300 & 540 & 9.51 & 7.4 & 17 & 521 \\
\hline 7 & 2,000 & 12 & 248 & 14 & 274 & 17 & 340 & 520 & 10.4 & 7.6 & 19 & 637 \\
\hline 8 & 2,700 & 7 & 156 & 21 & 556 & 16 & 330 & 600 & 16.2 & 8.8 & 20 & 599 \\
\hline 9 & 1,850 & 7 & 122 & 14 & 257 & 25 & 460 & 560 & 10.2 & 8.8 & 21 & \\
\hline 10 & 2,800 & 6 & 162 & 20 & 550 & 26 & 730 & 560 & 15.6 & 9.2 & 32 & 563 \\
\hline 11 & 2,400 & 6 & 130 & 7 & 350 & & & 580 & 12.2 & 9.8 & & \\
\hline
\end{tabular}

* Before operation the following values in milligrams in each $100 \mathrm{cc}$. were determined: serum bilirubin, 14.2; blood urea, 13.

indicates failure of recuperation by the liver and so is furthere vidence of the functional disturbance produced by the combined effect of the biliary obstruction and infection present in these cases.

In the cases in which marked cholerrhagia developed, the changes in the composition of the bile were most marked (tables 7,8 and 9, and figure $1 C$ and $D$ ). This supports the clinical impression that cholerrhagia is a symptom of serious hepatic dysfunction. In this condition the concentration and total output of bilirubin are somewhat reduced, 
whereas the reduction in the bile acids is even more striking. Surgeons (Judd and Lyons, 1923) have recognized that the presence of white bile in the biliary tract ordinarily is evidence of the accumulation within the gallbladder and biliary passages of mucus and of the secretion of the mucous membrane lining these ducts, and this conception has been amply confirmed by the experimental studies of Rous and McMaster (1921). A second variety of white bile has been recognized, on the other hand, as occasionally appearing after the

TABLE 9

Summary of data in case 9 (group 3)

\begin{tabular}{|c|c|c|c|c|c|c|c|c|c|c|c|c|}
\hline \multirow{3}{*}{$\begin{array}{l}\text { Days } \\
\text { after } \\
\text { opera- } \\
\text { tion }\end{array}$} & \multicolumn{9}{|c|}{ Bile } & \multirow{2}{*}{\multicolumn{3}{|c|}{ Blood seru }} \\
\hline & \multirow{2}{*}{$\begin{array}{l}\text { Vol- } \\
\text { ume }\end{array}$} & \multicolumn{2}{|c|}{ Bilirubin } & \multicolumn{2}{|c|}{$\begin{array}{c}\text { Bile acids as } \\
\text { glycocholic acid }\end{array}$} & \multicolumn{2}{|c|}{ Urea } & \multicolumn{2}{|c|}{$\begin{array}{l}\text { Chlorides as } \\
\text { sodium chloride }\end{array}$} & & & \\
\hline & & $\left|\begin{array}{c}\text { Concen- } \\
\text { tration }\end{array}\right|$ & Total & $\begin{array}{l}\text { Concen- } \\
\text { tration }\end{array}$ & Total & $\begin{array}{l}\text { Concen- } \\
\text { tration }\end{array}$ & Total & $\begin{array}{l}\text { Concen- } \\
\text { tration }\end{array}$ & Total & $\begin{array}{c}\text { Bili- } \\
\text { rubin }\end{array}$ & Urea & $\begin{array}{l}\text { Sodium } \\
\text { chloride }\end{array}$ \\
\hline & $c c$. & $\begin{array}{c}\text { per } 100 \\
\text { cc. }\end{array}$ & $m g m$. & $\begin{array}{c}\text { per } 100 \\
\text { cc. }\end{array}$ & $m g m$. & $\begin{array}{c}\text { mgm. } \\
\text { per } 100 \\
c c .\end{array}$ & $m g m$. & $\begin{array}{c}\text { mgm. } \\
\text { per } 100 \\
c c .\end{array}$ & $m g m$. & $\begin{array}{c}\text { per } 100 \\
c c .\end{array}$ & $\begin{array}{c}\text { mgm. } \\
\text { per } 100 \\
c c .\end{array}$ & $\begin{array}{c}\text { mgm. } \\
\text { per } 100 \\
c c .\end{array}$ \\
\hline 1 & 550 & 50 & 292 & 14 & 77 & 15 & 82 & 757 & 4.17 & & & \\
\hline 2 & 700 & 68 & 477 & 17 & 119 & 33 & 231 & 703 & 4.92 & & & \\
\hline 3 & 875 & 68 & 595 & 71 & 622 & 30 & 263 & 707 & 6.20 & 7.0 & 37 & \\
\hline 4 & 900 & 14 & 126 & 23 & 207 & 42 & 379 & 723 & 6.50 & & & 589 \\
\hline 5 & 1,400 & 16 & 224 & 22 & 308 & 39 & 546 & 740 & 10.35 & & & \\
\hline 6 & 1,700 & 16 & 274 & 19 & 323 & 56 & 952 & 730 & 12.40 & & & \\
\hline 7 & 1,825 & 16 & 292 & 27 & 493 & 80 & 1,460 & 707 & 12.90 & 5.0 & & \\
\hline 8 & 1,450 & 19 & 275 & 23 & 334 & 119 & 1,725 & 675 & 9.80 & & & \\
\hline 9 & 710 & 36 & 256 & 50 & 355 & 304 & 2,160 & 703 & 5.00 & & & \\
\hline 10 & 500 & 45 & 225 & 69 & 350 & 254 & 1,270 & 681 & 3.40 & 5.8 & & \\
\hline 11 & 760 & 36 & 374 & 71 & 540 & 263 & 2,000 & 707 & 5.38 & & 178 & 509 \\
\hline
\end{tabular}

* Before operation the following values in milligrams in each $100 \mathrm{cc}$. were determined: serum bilirubin, 12.9; blood urea, 16 .

institution of biliary drainage, and the data in case 7 illustrate the development of the latter condition. In this case the fluid secreted by the liver was almost completely acholic. Bile pigments were not present in as great a concentration as in the blood serum, whereas the sensitive Pettenkofer test showed the presence of but traces of bile acids. The characteristic constituents of the bile were entirely lacking, to all practical purposes, even though this deficient secretion was elaborated in considerable quantity. In this case, the development of 
hydrohepatosis had apparently progressed to a stage of almost complete abolition of the biliary functions of the liver.

In all of these cases the concentration of urea in the bile was approximately equal to that in the blood, and the daily output was, therefore, determined by the volume of the bile. In one patient (case 9 , figure $1 D$ ) postoperative renal insufficiency developed, and the concentration of urea in the blood increased to $300 \mathrm{mgm}$. in each 100 cc. The concentration of urea in the bile was increased in a similar manner. Marked cholerrhagia developed in this case at about the same time, with the result that the output of urea was increased to approximately 2 grams a day. Such an elimination of urea in the bile ordinarily would not be significant from the standpoint of renal function because the urea would be promptly reabsorbed on the passage of the bile into the intestine. In this case, however, the external drainage of the bile permitted vicarious elimination of the urea and consequent reduction in the burden borne by the kidneys.

Foster, Hooper and Whipple (1919) reported the development of abnormalities in the bones of dogs with prolonged external drainage of the bile. Only the excretion of chlorides in the bile was studied in the present series of cases. The concentration of chlorides in the bile was the same as, or slightly greater than, that in the blood serum, and was seemingly unaffected by such factors as changes in the food, the volume of the bile, or the intake of water or salt by the patient. The total output of chlorides in the bile varied between 1 and 4 grams. This is a quantity of salt that ordinarily would not be significant, although, if no salt were provided in the food, the loss might eventually become significant. The development of cholerrhagia, on the other hand, markedly increased the loss of chlorides; a maximal excretion of 16 grams in one day was observed and one patient lost 87 grams through the biliary tract in the course of a week. This is a quantity that would quickly lead to serious depletion of chlorides in the body if continued unchecked or unless the chlorides were replaced by the administration of appropriate amounts of chlorides, as was done in these cases.

In these cases oral administration of fluids was forced and an additional $1,000 \mathrm{cc}$. of a solution containing 100 grams of glucose and 10 grams of sodium chloride was given intravenously, so that the daily 
intake of fluids varied between 3,000 and $4,000 \mathrm{cc}$. Notwithstanding this liberal intake of fluids, the output of urine frequently was reduced following the onset of cholerrhagia. This is shown in table 4. Under these conditions, unless care is taken to secure an adequate intake, there is danger of serious depletion of both fluids and salt in consequence of such cholerrhagia.

It is recognized that experiments such as these are open to many criticisms. The collection of bile from a drainage tube, inserted in the gallbladder or common bile duct, is not always complete. When the drainage is through the gallbladder, possible effects of the normal concentrating action of that viscus cannot be excluded. The amount and character of the bile are not the same when there is complete external drainage as when the bile enters the intestine and the normal enterohepatic circulation is uninterrupted.

Most of these criticisms are inherent in the nature of the problem to be studied and cannot wholly be avoided. It is believed, nevertheless, that the changes reported are sufficiently distinct to indicate the response of the liver of man to the relief of biliary obstruction, and some of the modifications of that response caused by various associated conditions.

\section{SUMMARY}

The composition of the bile following the relief of biliary obstruction was studied in a series of nine cases.

The total daily output of bilirubin was more or less constant and was not related to the other factors studied in this series of cases. The concentration, on the other hand, varied inversely with the volume of the bile. One or two patients showed some evidence of a washing out of retained pigment, but if this occurred in all it took place so slowly as not to be apparent in observations of as short duration as these.

Biliary obstruction inhibits or stops the formation of bile acids. If the liver is not too greatly injured there is a relatively rapid return to normal; otherwise the return is greatly delayed. This was true both with regard to the concentration and total amount of bile acids.

The concentration of chlorides in the bile is slightly greater than that in the blood serum. With cholerrhagia, the resultant loss of 
salts becomes so great as to be of clinical significance. The loss of fluids by this channel may also be so great as to cause diminution in the output of urine.

The concentration of urea in the bile apparently varies directly with that in the blood. Ordinarily this pathway of elimination is not significant, but in one case there was considerable loss of urea through the fistula.

\section{BIBLIOGRAPHY}

1. Aldrich, Martha, and Bledsoe, Mary Sue, J. Biol. Chem., 1928, lxxvii, 519. Studies in the Metabolism of the Bile. I. A Quantitative Pettenkofer Test Applicable to the Determination of Bile Acids in the Blood.

2. Bidder, F., and Schmidt, C., Die Verdauungssäfte und der Stoffwechsel eine physiologischchemische Untersuchung. Mittau, G. A. Reyher, 1852, 413 pp.

3. Brakefield, J. L., and Schmidt, C. L. A., J. Biol. Chem., 1926, lxvii, 523. Studies on the Synthesis and Elimination of Certain Bile Components in Obstructive Jaundice.

4. Chabrol, E., Bénard, H., and Bariéty, M., Bull. et mém. Soc. méd. d. hôp. de Par., 1926, 1, 992. Etudes comparatives des pigments, des sels biliares et de la cholestérine dans un cas de fistule du cholédogue (1).

5. von Czyhlarz, Ernst, Fuchs, Adolf, and von Fürth, Otto, Biochem. Ztschr., 1913, xlix, 120. Über die analytische Zusammensetzung der menschlichen Galle.

6. Foster, M. G., Hooper, C. W., and Whipple, G. H., J. Biol. Chem., 1919, xxxviii, 367. The Metabolism of Bile Acids. II. Normal Fluctuations in Healthy Bile Fistula Dogs.

7. Foster, M. G., Hooper, C. W. and Whipple, G. H., J. Biol. Chem., 1919, xxxviii, 393. The Metabolism of Bile Acids. IV. Endogenous and Exogenous Factors.

8. Greene, C. H., and Snell, A. M., J. Biol. Chem., 1928, lxxviii, 691. Studies in the Metabolism of the Bile. II. The Sequence of Changes in the Blood and Bile Following the Intravenous Injection of Bile or Its Constituents.

9. Greene, C. H., Snell, A. M., and Walters, Waltman, Arch. Int. Med., 1925, xxxvi, 248. Diseases of the Liver. I. A Survey of Tests for Hepatic Function.

10. Hawk, P. P., and Bergeim, Olaf, Practical Physiological Chemistry. Philadelphia, P. Blakiston's Son and Co., 1926, 9th ed. pp. 374-402.

11. Judd, E. S., and Lyons, J. H., Ann. Surg., 1923, lxxvii, 281. White Bile in the Common Duct.

12. McClure, C. W., Mendenhall, W. L., and Huntsinger, Mildred E., J. Am. Med. Assoc., 1925, lxxxv, 1537. The Evaluation and Treatment of Disturbed Liver Function. 
13. McMaster, P. D., J. Exp. Med., 1924, xl, 25. Studies on the Total Bile. VI. The Influence of Diet upon the Output of Cholesterol in the Bile.

14. McMaster, P. D., Broun, G. O. and Rous, Peyton, J. Exp. Med., 1923; xxxvii, 395. Studies on the Total Bile. I. The Effects of Operation, Exercise, Hot Weather, Relief of Obstruction, Intercurrent Disease, and Other Normal and Pathological Influences. J. Exp. Med., 1923, xxxvii, 685. III. On the Bile Changes Caused by a Pressure Obstacle to Secretion; and on Hydrohepatosis.

15. McNee, J. W., Quart. J. Med., 1914, vii, 221. Cholesterin: An Account of its Relations to Pathology and Physiology.

16. Rosenthal, F., and von Falkenhausen, M., Klin. Wchnschr., 1923, ii, 1487. Weitere Beiträge zur Physiologie und Pathologie der Gällensäurensekretion beim Menschen. III. Mitteilung.

17. Rosenthal, F., von Falkenhausen, M., and Freund, H., Arch. f. exper. Path. u. Pharm., 1926, cxi, 170. Weitere Beiträge zur Physiologie und Pathologie de Gallensäurensekretion beim Menschen. V. Utber das Phänomen der Umkehr der Gallensäurenrelationen in der Galle von Leberkranken.

18. Rous, Peyton, Am. J. Med. Sci., 1925, clxx, 625. The Biliary Aspects of Liver Disease.

19. Rous, Peyton, and McMaster, P. D., J. Exp. Med., 1921, xxxiv, 47. The Concentrating Activity of the Gallbladder.

20. Rous, Peyton, and McMaster, P. D., J. Exp. Med., 1921, xxxiv, 75. Physiological Causes for the Varied Character of Stasis Bile.

21. Schiff, M., Arch. f. d. ges. Physiol., 1870, iii, 598. Bericht über einige Versuchsreihen.

22. Smyth, F. S., and Whipple, G. H., J. Biol. Chem., 1924, lix, 623. Bile Salt Metabolism. I. Influence of Chloroform and Phosphorus on Bile Fistula Dogs.

23. Snell, A. M., Greene, C. H., and Rowntree, L. G., Arch. Int. Med., 1927, xl, 471. Diseases of the Liver. VII. Further Studies in Experimental Obstructive Jaundice.

24. Stadelmann, Ernst, Der Icterus und seine verschiedenen Formen. Nebst Beiträgen zur Physiologie und Pathologie der Gallensecretion. Stuttgart, F. Enke, 1891, 287 pp.

25. Stadelmann, E., Ztschr. f. Biol., 1896, xxxiv, 1. Ueber den Kreislauf der Galle im Organismus.

26. Walters, Waltman, Greene, C. H., and Fredrickson, C. H., Ann. Surg., 1930, xci, 686. The Composition of the Bile Following the Relief of Biliary Obstruction: Report of a Series of Illustrative Cases.

27. Walters, Waltman, and Parham, Duncan, Surg., Gynec., and Obst., 1922, $x x x v, 605$. Renal and Hepatic Insufficiency in Obstructive Jaundice.

28. Whipple, G. H., Physiol. Rev., 1922, ii, 440. The Origin and Significance of the Constituents of the Bile.

29. Wisner, F. P., and Whipple, G. H., Am. J. Physiol., 1922, lx, 119. Variations in Output of Bile Salts and Pigments During 24-hour Periods; Observations on Standard Bile Fistula Dogs. 